\title{
Letramento funcional em saúde na terapia renal substitutiva: revisão integrativa
}

Functional health literacy in renal replacement therapy: an integrative review Alfabetización funcional en salud en terapia de reemplazo renal: revisión integradora

Késia Tomasi da Rocha ${ }^{1}$ id https://orcid.org/0000-0001-7479-3494 Ana Elizabeth Figueiredo ${ }^{1}$ id https://orcid.org/0000-0002-8555-8649

\section{Como citar:}

Rocha KT, Figueiredo AE. Letramento funcional em saúde na terapia renal substitutiva: revisão integrativa. Acta Paul Enferm. 2020;33:eAPE20180124.

DOI http://dx.doi.org/10.37689/actaape/2020RI0124

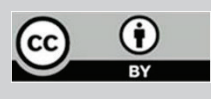

Descritores

Alfabetização em saúde; Doença renal crônica; Diálise peritoneal; Transplante renal; Educação em saúde

Keywords

Health Literacy: Renal insufficiency, chronic; Peritoneal dialysis; Kidney transplantation; Health Education

\section{Descriptores} Alfabetización en salud; Insuficiencia renal crónica; Diálisis peritoneal; Trasplante de riñón; Educación en salud

\section{Submetido \\ 21 de Junho de 2018 \\ Aceito \\ 4 de Novembro de 2019}

\section{Autor correspondente}

Késia Tomasi da Rocha E-mail: trkesia@gmail.com.

\section{Resumo}

Objetivo: Analisar os instrumentos disponíveis para a identificação do grau de letramento funcional em saúde dos pacientes submetidos a terapia renal substitutiva.

Métodos: Trata-se de uma revisão integrativa, realizada nas bases de dados PubMed, SciELO, CINAHL e Web of Science, entre outubro de 2017 a janeiro de 2018. Foram incluídos artigos originais completos, disponíveis a partir do ano de 2010 até 0 momento da busca e nos idiomas inglês, espanhol e português. Os descritores utilizados foram: hemodialysis, peritoneal dialysis, transplantation, renal dialysis patient information e health literacy.

Resultados: Dos 4.286 estudos encontrados na busca, 16 foram incluídos para análise. Doze instrumentos diferentes foram utilizados, sendo as ferramentas mais aplicadas o Rapid Estimate Adult Literacy of Medicine (REALM), utilizado em quatro pesquisas e o Newest Vital Sign (NVS), usado em três estudos. Doze instrumentos foram aplicados em pacientes submetidos a hemodiálise, três nos indivíduos em diálise peritoneal e foram encontrados três instrumentos específicos para transplantados renais. As ferramentas mais atuais têm avaliado aspectos sociais, autocuidado e capacidade de gerenciamento e entendimento dos pacientes em terapia renal substitutiva.

Conclusão: Os doze instrumentos para mensuração do letramento funcional em saúde para uso em pacientes em terapia renal substitutiva apresentam propriedades psicométricas boas, porém, apenas um está validado no Brasil. Ressalta-se a lacuna de uso de ferramentas que avaliem o letramento nos pacientes em diálise peritoneal.

\section{Abstract}

Objective: To analyze the instruments available to identify the functional health literacy level of patients submitted to renal replacement therapy.

Methods: Integrative review carried out by searching publications in the PubMed, Scientific Electronic Library Online, Cumulative Index to Nursing and Allied Health Literature, and Web of Science databases between October 2017 and January 2018. Full original articles in English, Spanish, or Portuguese made available from 2010 to the moment the search was performed were included. The descriptors used in the search were hemodialysis, peritoneal dialysis, transplantation, renal dialysis patient information, and health literacy.

Results: Sixteen out of the 4,286 studies found were included to be analyzed. It was observed that 12 different instruments were used, with the Rapid Estimate of Adult Literacy in Medicine and the Newest Vital Sign being the most frequently used tools, applied in four and three studies, respectively. Twelve instruments were applied in patients submitted to hemodialysis, three in those who underwent peritoneal analysis, and three were 
specific to patients who went through kidney transplant. The most recent tools have assessed social aspects, self-care, and management and understanding capacity in patients under renal replacement therapy.

Conclusion: The twelve instruments to measure functional health literacy in patients under renal replacement therapy show satisfactory psychometric properties, but only one is validated to be used in Brazil. The insufficient use of tools to evaluate literacy in patients undergoing peritoneal dialysis was noteworthy.

\section{Resumen}

Objetivo: Analizar los instrumentos disponibles para identificar el nivel de alfabetización funcional en salud de los pacientes que realizan terapia de reemplazo renal.

Métodos: Se trata de una revisión integradora, realizada en las bases de datos PubMed, SciELO, CINAHL y Web of Science, entre octubre de 2017 y enero de 2018. Se incluyeron artículos originales completos, disponibles a partir de 2010 hasta el momento de la búsqueda, en los idiomas inglés, español y portugués. Los descriptores utilizados fueron: hemodialysis, peritoneal dialysis, transplantation, renal dialysis patient information y health literacy.

Resultados: De los 4.286 estudios encontrados en la búsqueda, se incluyeron 16 en el análisis. Se utilizaron 12 instrumentos diferentes y las herramientas más aplicadas fueron Rapid Estimate Adult Literacy of Medicine (REALM), utilizado en cuatro estudios, y Newest Vital Sign (NVS), utilizado en tres estudios. Se aplicaron 12 instrumentos a pacientes que realizaban hemodiálisis, tres a individuos en diálisis peritoneal y se encontraron tres instrumentos específicos para trasplantados renales. Las herramientas más actuales evaluaron aspectos sociales, autocuidado y capacidad de gestión y comprensión de los pacientes en terapia de reemplazo renal.

Conclusión: Los 12 instrumentos para medir la alfabetización funcional en salud de pacientes en terapia de reemplazo renal presentaron propiedades psicométricas buenas, pero solo uno está validado en Brasil. Se destaca un vacío en el uso de herramientas que evalúen la alfabetización de pacientes en diálisis peritoneal.

\section{Introdução}

Crescente na população mundial, a doença renal crônica (DRC) está associada ao aumento no risco para admissóes hospitalares e morbidades, contribuindo significativamente para todas as causas de morte cardiovasculares. ${ }^{(1,2)}$ Estima-se que mais de 2 milhôes de indivíduos em todo o mundo sejam submetidos a diálise ou transplante renal, porém este número pode representar apenas $10 \%$ de todos os pacientes que realmente necessitam destes tratamentos para sobreviver. ${ }^{(3)}$

No período de 2007 a 2016, entre os pacientes em terapia renal substitutiva (TRS) no Brasil, a incidência de indivíduos em diálise aumentou em aproximadamente $37 \%$. ${ }^{(4)}$ Cerca de $92,1 \%$ dos pacientes realizam a substituição da função renal por hemodiálise (HD), 7,9\%, por diálise peritoneal (DP) e cerca de 29.000 indivíduos estão em lista de espera para transplante renal (TxR). ${ }^{(4)}$

Dada a prevalência e incidência da DRC na população, bem como a complexidade de seu tratamento, a tomada de decisão compartilhada e a gestão do autocuidado são aspectos imprescindíveis para o êxito nos desfechos clínicos destes indivíduos. ${ }^{(5,6)}$ Estes fatores podem ser consideravelmente influenciados pelo nível de Letramento Funcional em Saúde (LFS) ou Alfabetização em Saúde, o qual consiste na capacidade do indivíduo de obter, processar e compreender as informaçóes e os serviços básicos de saúde necessários para a tomada de decisôes pertinentes sobre a sua própria saúde e cuidados médicos. ${ }^{(6,7)}$

A alfabetização e o letramento são conceitos indissociáveis do ponto de vista educacional. A alfabetização é tornar o indivíduo capaz de ler e escrever, enquanto o letramento se refere às habilidades de uso deste sistema em atividades de leitura e escrita. ${ }^{(8)} \mathrm{O}$ termo "funcional", acrescido ao letramento, é relativo à capacidade do indivíduo de utilizar a leitura, escrita e cálculo sempre que for preciso realizar determinada atividade ou construir novos aprendizados necessários ao seu desenvolvimento pessoal e contexto social. ${ }^{(9)}$

Uma recente metanálise demonstrou que o LFS inadequado $(43,47 \%)$ foi mais prevalente entre portadores de Diabetes mellitus ${ }^{(10)}$ e, entre os pacientes com DRC ( $n=137), 26 \%$ apresentaram LFS limitado. ${ }^{(1)}$ Tal achado, em outras pesquisas, está associado com a pior qualidade de vida, ${ }^{(12)}$ menor adesão ao tratamento medicamentoso, ${ }^{(13)}$ falta de assiduidade nas consultas médicas ${ }^{(14)}$ e maior taxa de hospitalização, ${ }^{(14)}$ impactando desfavoravelmente no desfecho clínico destes indivíduos.

Para que o enfermeiro avalie o nível de LFS e selecione o resultado de suas intervençóes, são necessários instrumentos específicos e validados para o idioma e realidade brasileira. Desta forma, identificar as ferramentas disponíveis, conhecer suas carac- 
terísticas e propriedades psicométricas pode direcionar a conduta de seleção para o seu uso na prática clínica e fomentar pesquisas futuras, de tradução e adaptação transcultural.

O objetivo deste estudo é analisar os instrumentos disponíveis para a identificação do grau de LFS na população submetida a terapia de substituiçáo da função renal.

\section{Métodos}

Estudo realizado a partir de uma revisão integrativa da literatura, a qual permite abordar pesquisas qualitativas e/ou quantitativas, tendo a capacidade de explorar diversos métodos cujas conclusóes, quando avaliadas de maneira sistemática e ordenada, potencialmente podem ser aplicadas à prática clínica. ${ }^{(15)}$

Para elaboração deste estudo foram seguidas as seguintes etapas: identificação do tema e seleção da hipótese ou questão de pesquisa para a elaboração da revisão integrativa, estabelecimento de critérios para inclusão e exclusão de estudos/amostragem ou busca na literatura, definiçãao das informaçóes a serem extraídas dos estudos selecionados/categorização dos estudos, avaliação dos estudos incluídos na revisão integrativa, interpretação dos resultados, apresentação da revisão/síntese do conhecimento. ${ }^{(16)}$

Os critérios de inclusão para a busca e seleção das publicaçóes foram: artigos originais completos, disponíveis na íntegra nas bases de dados a partir do ano de 2010 (período em que o descritor "alfabetização em saúde" se tornou disponível) até o momento da busca e nos idiomas inglês, espanhol e português. Os estudos deveriam estar indexados em pelo menos uma das bases de dados: PubMed, Cumulative Index to Nursing and Allied Health Literature (CINAHL), Scientific Electronic Library Online (SciELO) e Web of Science (WOS).

Conforme a terminologia dos Descritores em Ciências da Saúde (DeCS), os estudos deveriam ser localizáveis pelos seguintes termos: hemodialysis, peritoneal dialysis, transplantation, renal dialysis patient information e health literacy. A fim de sistematizar a busca na literatura, foram utilizados os operadores booleanos peritoneal dialysis or renal dialysis or transplantation or hemodialysis and health literacy or patient education. Foram excluídas as publicaçóes repetidas nas bases de dados, bem como as teses, dissertaçóes, monografias e aqueles em que constava apenas o resumo.

A coleta dos dados ocorreu entre outubro de 2017 e janeiro de 2018 e foi realizada por duas pesquisadoras independentes que, quando da seleçáo dos artigos, primeiramente avaliaram o título, seguindo-se o resumo e, posteriormente, fizeram a leitura do estudo na íntegra.

\section{Resultados}

Os instrumentos que avaliam o LFS podem ser gerais ou específicos e, neste estudo, buscou-se analisar na literatura científica as ferramentas disponíveis para pacientes com DRC em TRS. Assim, foram encontradas 4.286 publicaçóes e selecionados 16 artigos para análise (Quadro 1). Ressalta-se que a base de dados SciELO apresentou apenas três publicaçóes, que não eram pertinentes ao tema desta revisão.

Quadro 1. Seleção dos artigos científicos para a revisão integrativa

\begin{tabular}{|l|c|c|c|c|}
\hline $\begin{array}{l}\text { Pesquisa } \\
\text { nas bases de } \\
\text { dados }\end{array}$ & \multicolumn{4}{|c|}{ Publicações: 4.286 } \\
\hline $\begin{array}{l}\text { Aplicação } \\
\text { dos critérios } \\
\text { de inclusão/ } \\
\text { exclusão }\end{array}$ & $\begin{array}{c}\text { Não aplicáveis } \\
\text { à questão } \\
\text { norteadora } \\
\text { deste estudo: } \\
4.243\end{array}$ & $\begin{array}{c}\text { Repetido em } \\
\text { outra base de } \\
\text { dados: } 18\end{array}$ & $\begin{array}{c}\text { Excluídos por } \\
\text { serem carta ao } \\
\text { editor e apenas } \\
\text { resumos: } 7\end{array}$ & $\begin{array}{c}\text { Não disponíveis } \\
\text { na íntegra: } 2\end{array}$ \\
\hline $\begin{array}{l}\text { Total de artigos } \\
\text { selecionados }\end{array}$ & \multicolumn{4}{|c|}{ PubMed: 16 artigos } \\
\hline
\end{tabular}

Doze instrumentos diferentes foram utilizados para avaliar o nível de LFS nos pacientes submetidos a TRS, os quais seguem descritos no quadro 2 .

Quanto aos locais de realização dos estudos, a maioria ocorreu nos Estados Unidos da América $(\mathrm{n}=11)$, seguidos da Austrália $(\mathrm{n}=2)$, Canadá $(\mathrm{n}=1)$, Cingapura $(n=1)$ e Taiwan $(n=1)$. Observa-se que o primeiro estudo publicado sobre a temática foi no ano de $2010^{(28)}$ e, no período de 2015 , houve um aumento na frequência das publicaçóes sobre este tema. ${ }^{(19,21,22,25)}$

Em relação ao nível de evidência, ${ }^{(32)} 12$ estudos foram considerados nível $6,{ }^{(17-28)}$ dos quais dois se re- 


\section{Quadro 2. Instrumentos de avaliação do Letramento Funcional em Saúde de pacientes em terapia renal substitutiva}

\begin{tabular}{|c|c|c|c|}
\hline Autor(es) & Instrumento & Aspectos avaliados/classificação & Propriedades psicométricas conhecidas \\
\hline Demian MN, et al. ${ }^{(17)}$ & \multirow{2}{*}{$\begin{array}{l}\text { Health Literacy } \\
\text { Questionnaire } \\
\text { (HLQ) }\end{array}$} & \multirow[b]{2}{*}{$\begin{array}{l}\text { Avalia habilidades necessárias para o letramento em saúde: compreensão, apoio e vínculo } \\
\text { com os profissionais de saúde; gerenciamento da própria saúde; apoio social; navegação pelo } \\
\text { sistema de saúde; capacidade de compreensão das informações para tomada de decisão sobre } \\
\text { sua saúde. } \\
\text { Classificação: } 44 \text { itens, divididos em nove domínios distintos, cada um com 4-6 graus de } \\
\text { concordância em uma escala do tipo Likert. Pontuações mais altas indicam melhor letramento } \\
\text { em saúde. }\end{array}$} & \multirow[t]{2}{*}{ Alfa de Cronbach 0,77 - 0,90 } \\
\hline Dodson S, et al..$^{(18)}$ & & & \\
\hline Lambert K, et al. ${ }^{(19)}$ & $\begin{array}{l}\text { Health Literacy } \\
\text { Management Scale } \\
\text { (HeLMS) }\end{array}$ & $\begin{array}{l}\text { Avalia habilidades necessárias para o letramento em saúde: atitudes e proatividade do paciente } \\
\text { em relação à sua saúde; compreensão das informações de saúde; suporte social; fatores } \\
\text { socioeconômicos para o acesso aos serviços de saúde; acesso ao atendimento médico clínico- } \\
\text { geral; comunicação com profissionais de saúde e uso das informações de saúde. } \\
\text { Classificação: } 29 \text { itens, divididos em oito domínios. As respostas são pontuadas em uma escala } \\
\text { Likert de cinco pontos e dicotomizadas como sem dificuldade (pontuação de } 5 \text { na escala) ou } \\
\text { qualquer dificuldade (pontuação de } 1 \text { a } 4 \text { na escala). Pontuações mais altas indicam melhor } \\
\text { letramento em saúde. }\end{array}$ & Alfa de Cronbach 0,82 - 0,89 \\
\hline Chiu $\mathrm{CH}$, et al. ${ }^{(20)}$ & $\begin{array}{l}\text { Alfabetização em saúde } \\
\text { em Chinês }\end{array}$ & $\begin{array}{l}\text { Apresenta } 52 \text { itens, divididos em duas seções: alfabetização em saúde (dividida em sete sub- } \\
\text { construtos de alfabetização: funcional, comunicativa, interativa, crítica, conhecimentos básico e } \\
\text { avançado de saúde e segurança do paciente) e dados demográficos. } \\
\text { Classificação: } 1 \text { ponto por resposta correta das quatro opções de múltipla escolha, sendo a } \\
\text { pontuação máxima } 26 \text { e a pontuação mínima zero. Pontuações mais altas indicam melhor } \\
\text { letramento em saúde. }\end{array}$ & Alfa de Cronbach $\cong 0,81$ \\
\hline Cavanaugh KL, et al..(21) & $\begin{array}{l}\text { Brief Health Literacy Screen } \\
\text { (BHLS) }\end{array}$ & $\begin{array}{l}\text { Consiste em três perguntas que avaliam a autoconfiança do paciente, a frequência com que } \\
\text { precisa de ajuda para ler documentos referentes à sua saúde e compreensão de sua condição } \\
\text { de saúde. } \\
\text { Classificação: Cada questão pode receber até } 5 \text { pontos, que são somados para produzir uma } \\
\text { pontuação total de } 3 \text { a 15, sendo considerados letramento limitado (3-9 pontos) e letramento } \\
\text { adequado (10-15). }\end{array}$ & $\begin{array}{l}\text { A área sob a curva Receiver Operating } \\
\text { Characteristic (ROC), respectivamente, } \\
\text { para cada questão foi } 0,87,0,80 \text { e } 0,76 \text {. }\end{array}$ \\
\hline Kazley AS, et al.(22) & $\begin{array}{l}\text { Rapid Estimate of } \\
\text { Adult Literacy of Medicine- } \\
\text { Transplant } \\
\text { (REALM-T) }\end{array}$ & $\begin{array}{l}\text { Apresenta } 69 \text { palavras, as quais devem ser corretamente pronunciadas, em voz alta, pelo } \\
\text { paciente. } \\
\text { Classificação: letramento em saúde adequado: } 60 \text { a 69; letramento marginal: } 45 \text { a 59; e } \leq 44 \\
\text { letramento inadequado. }\end{array}$ & Alfa de Cronbach $\cong 0,94$ \\
\hline Kazley AS, et al.(23) & \multirow{2}{*}{$\begin{array}{l}\text { Newest Vital Sign } \\
\text { (NVS) }\end{array}$} & \multirow{2}{*}{$\begin{array}{l}\text { Consiste em duas prescrições médicas e três questões sobre cada uma delas, que levam } 0 \\
\text { paciente a ler, entender e buscar informações. } \\
\text { Classificação: letramento adequado: de } 4 \text { a 6; letramento marginal: } 2 \text { a 3; e de } 1 \text { a } 0 \text { letramento } \\
\text { inadequado. }\end{array}$} & \multirow{2}{*}{$\begin{array}{l}\text { Alfa de Cronbach }>0,76 \text { para o } \\
\text { instrumento em inglês e } 0,69 \text { para a } \\
\text { ferramenta em espanhol. }\end{array}$} \\
\hline Escobedo W, et al. ${ }^{(24)}$ & & & \\
\hline Kazley AS, et al. ${ }^{(22)}$ & \multirow{2}{*}{$\begin{array}{l}\text { Decision-Making Capacity } \\
\text { Assessment Tool } \\
\text { (DMCAT) }\end{array}$} & \multirow{2}{*}{$\begin{array}{l}\text { Apresenta seis perguntas que avaliam o conhecimento e compreensão da diálise e o processo } \\
\text { de transplante. } \\
\text { Classificação: } 3 \text { pontos por resposta correta, sendo } 18 \text { o escore máximo. Pontuações mais altas } \\
\text { indicam melhor conhecimento. }\end{array}$} & \multirow{2}{*}{$\begin{array}{l}\text { Coeficiente de correlação de Pearson entre } \\
0,56 \text { - } 0,73 \text { e a confiabilidade teste- } \\
\text { reteste e entre os avaliadores foi aceitável } \\
(0,65) \text {. }\end{array}$} \\
\hline Kazley AS, et al.(23) & & & \\
\hline Jain D, et al. ${ }^{(25)}$ & \multirow{4}{*}{$\begin{array}{l}\text { Rapid Estimate of } \\
\text { Adult Literacy of Medicine } \\
\text { (REALM) }\end{array}$} & \multirow{4}{*}{$\begin{array}{l}\text { Apresenta } 66 \text { palavras, as quais devem ser corretamente pronunciadas, em voz alta, pelo } \\
\text { paciente. } \\
\text { Classificação: letramento em saúde adequado: } 60 \text { a 66; letramento marginal: } 45 \text { a 59; e } \leq 44 \\
\text { letramento inadequado. }\end{array}$} & \multirow{4}{*}{$\begin{array}{l}{ }^{*} \text { Correlaciona-se bem com outros } \\
\text { testes de alfabetização e possui alta } \\
\text { confiabilidade teste-reteste. }\end{array}$} \\
\hline Green JA, et al.,;, (26) & & & \\
\hline Green JA, et al..$^{(27)}$ & & & \\
\hline Cavanaugh KL, et al.(28) & & & \\
\hline Lai AY, et al. ${ }^{(29)}$ & $\begin{array}{l}\text { Functional, Communicative } \\
\text { and Critical Health Literacy } \\
\text { (FCCHL) }\end{array}$ & $\begin{array}{l}\text { Apresenta } 14 \text { itens, sendo cinco itens de letramento funcional (avalia habilidades básicas } \\
\text { de leitura e escrita) e comunicativo (capacidade de extrair informações de variados meios } \\
\text { de comunicação) e quatro itens de letramento crítico (capacidade de avaliar criticamente as } \\
\text { informações sobre a sua própria saúde). } \\
\text { Classificação: Escala Likert, pontuada de } 1 \text { a } 4 \text { (nunca a frequentemente) para cada item. Os } \\
\text { escores dos itens de cada subescala foram somados e divididos pelo número de itens } \\
\text { constituintes da subescala. Pontuações mais altas indicam melhor letramento em saúde. }\end{array}$ & $\begin{array}{l}0 \text { Alfa de Cronbach para os letramentos } \\
\text { funcional, comunicativo e crítico foi, } \\
\text { respectivamente, 0,84, 0,77 e 0,65. }\end{array}$ \\
\hline \multirow[t]{2}{*}{ Brice $\mathrm{JH}$, et al. ${ }^{(30)}$} & $\begin{array}{l}\text { Single item literacy } \\
\text { screener } \\
\text { (SILS) }\end{array}$ & $\begin{array}{l}\text { Consiste em uma pergunta, que avalia o entendimento do paciente quando lê materiais } \\
\text { relacionados à sua saúde. } \\
\text { Classificação: Escala Likert de } 1 \text { a 5, variando de nunca para sempre. Pontuações mais altas } \\
\text { indicam melhor letramento em saúde. }\end{array}$ & $\begin{array}{l}\text { A área sob a curva ROC, foi de } 0,67(0,60 \\
\text { a } 0,74) \text {. }\end{array}$ \\
\hline & $\begin{array}{l}\text { Two-item literacy screener } \\
\text { (TILS) }\end{array}$ & $\begin{array}{l}\text { Consiste em duas perguntas, que avaliam a capacidade/frequência de leitura de diferentes } \\
\text { materiais. } \\
\text { Classificação: Escala Likert, de excelente a péssima. }\end{array}$ & $\begin{array}{l}\text { A área sob a curva ROC, foi de } 0,66(0,59 \\
\text { a } 0,73) \text {. }\end{array}$ \\
\hline Brice JH, et al; ${ }^{(30)}$ & \multirow{2}{*}{$\begin{array}{l}\text { Short Test of Functional } \\
\text { Health Literacy in Adults } \\
\text { (S-TOFHLA) }\end{array}$} & \multirow{2}{*}{$\begin{array}{l}\text { Apresenta } 36 \text { lacunas, em que o paciente deve escolher, dentre quatro opções, a palavra que } \\
\text { mais se enquadra ao contexto da frase - objetiva avaliar a compreensão de leitura; e quatro } \\
\text { cartões (prescrição médica, marcação de consulta) que avaliam a capacidade de numeramento. } \\
\text { Classificação: Considera-se letramento inadequado: } 0 \text { a 16; marginal: } 17 \text { a } 22 ; \text { e de } 23 \text { a 36, } \\
\text { adequado. }\end{array}$} & \multirow{2}{*}{$\begin{array}{l}0 \text { Alfa de Cronbach foi de } 0,68 \text { para os } 36 \\
\text { itens de leitura e } 0,97 \text { para as imagens do } \\
\text { instrumento. }\end{array}$} \\
\hline Adeseun GA, ${ }^{(31)}$ & & & \\
\hline
\end{tabular}

*Artigo indisponivel para a avaliação dos dados psicométricos

ferem à elaboração de instrumentos de mensuração do letramento, aplicados em pacientes submetidos a $\mathrm{HD}^{(20)}$ e transplantados renais. ${ }^{(24)}$ Quatro pesquisas apresentam nível 4, sendo estas os estudos de coor- te, os quais visam avaliar os desfechos de pacientes em TRS de acordo com o nível de LFS. ${ }^{(29-31,33)}$

Quanto ao idioma das escalas, a maioria das ferramentas consta com o seu original em inglês, 
com ausência do processo de validação e adaptação transcultural para outras línguas. As ferramentas $\mathrm{NVS}^{(23,24)}$ e DMCAT ${ }^{(22,23)}$ estão disponíveis também em espanhol e o instrumento REALM, ${ }^{(25-28)}$ além destes dois idiomas, também está validado para o português do Brasil. ${ }^{(34)}$

Ressalta-se que o instrumento $\mathrm{BHLS}^{(21)}$ foi utilizado também por Dageforde e cols., ${ }^{(33)}$ porém com a denominação de Short Literacy Screen (SLS). Trata-se da mesma ferramenta, no entanto, os pontos de corte para a classificação do letramento diferem entre estas publicaçóes. Cavanaugh e cols. ${ }^{(21)}$ utilizaram a pontuação do instrumento conforme descrita na publicação de validação do mesmo.

Referente às ferramentas utilizadas, o REALM foi o primeiro com o qual se avaliou o letramento nesta população, no ano de $2010,{ }^{(28)}$ e foi aplicado em mais três pesquisas encontradas. ${ }^{(25-27)}$ Quanto aos estudos mais recentes, os dois últimos foram publicados em 2016 e se utilizaram da ferramenta HLQ, a qual busca avaliar também a subjetividade do paciente quanto às informaçóes recebidas nos serviços de saúde. ${ }^{(17,18)}$

Destaca-se que foram encontradas três ferramentas específicas que avaliam o grau de LFS em pacientes transplantados renais, sendo: o NVS versão adaptada para transplante, o qual consiste na interpretação de duas prescrições médicas; ${ }^{(22)} \mathrm{o}$ REALM-T, que avalia a leitura e pronúncia de 69 termos da área da saúde; ${ }^{(22)}$ e o DMCAT, cuja proposta é avaliar a capacidade do paciente em reconhecer os sintomas da DRC terminal e o processo de diálise e transplante. ${ }^{(22,23)}$

\section{Discussão}

Foram identificados neste estudo 12 instrumentos que têm sido utilizados para avaliação do letramento dos pacientes em TRS. Tais ferramentas, inicialmente, tinham a premissa de avaliar apenas a capacidade numérica e/ou de compreensão de leitura do paciente, porém os estudos mais atuais apresentam instrumentos que avaliam aspectos gerais do indivíduo, como a compreensão sobre a sua doença e tramitação nos serviços de saúde. ${ }^{(17,19,30)}$
Referente aos instrumentos utilizados para avaliação de pacientes em diálise, com exceção da ferramenta SLS, todas as outras já foram aplicadas em pacientes submetidos a HD, possivelmente por esta ser a terapia mais prevalente e incidente entre os indivíduos com DRC dialítica. Quanto aos pacientes em DP, apenas três dos 12 instrumentos encontrados foram aplicados neste grupo, o que reflete a carência de estudos com esta população, salientando-se, no entanto, que este é o método em que o paciente mais depende do seu entendimento e autocuidado. ${ }^{(18,19,25)}$

A ferramenta REALM, mais frequentemente utilizada, foi desenvolvida nos anos de 1990 com o objetivo de ser um instrumento de triagem rápida na identificação de pacientes com habilidades de leitura limitadas. Apesar de outras ferramentas terem surgido posteriormente, várias são baseadas neste modelo, o qual se limita apenas à leitura e pronúncia de termos, sem considerar que o paciente pode se tornar apenas um mero reprodutor de vocábulos que comumente ouve no serviço de saúde.

O primeiro estudo que avaliou o LFS em pacientes com Tx renal foi publicado em 2013, utilizando-se da ferramenta NVS, a qual primariamente foi desenvolvida baseada em seis questóes sobre uma etiqueta nutricional de sorvete. ${ }^{(24)}$ Embora compreender orientaçóes médicas escritas faça parte do LFS, outros aspectos como o conhecimento da doença, apoio social e tramitação nos serviços de saúde não são avaliados neste instrumento, enquanto na ferramenta DMCAT é considerado apenas o conhecimento quanto à diálise e ao processo de transplante. ${ }^{(22,23)}$

Atualmente, destaca-se a importância de tratar a pessoa em seu processo de doença holisticamente, incluindo não apenas a comprenssão das informaçôes que recebe, como também o vínculo com os profissionais de saúde, o seu autocuidado, apoio social, entre outros aspectos. ${ }^{(18)}$ Como demonstrado nas publicaçóes mais recentes, a tendência é que os instrumentos de avaliação do LFS estejam abrangendo os aspectos citados acima, já encontrados nas ferramentas $\mathrm{HLQ}^{(17,18)}$ e HeLMS. ${ }^{(19)}$

O HLQ é um questionário de autorrelato, com 44 itens, divididos em nove domínios e classificados 
por uma escala do tipo Likert de 4 pontos. ${ }^{(35)}$ Embora não tenha sido validado especificamente na população portadora de doença renal, tal processo deu-se com pacientes que apresentavam outras condiçóes crônicas (cardiopatia e diabetes). ${ }^{(17)}$ Esse instrumento avalia a capacidade do gerenciamento ativo da saúde, apoio social à minha saúde, avaliação da informação em saúde, capacidade de interagir ativamente com profissionais de saúde e navegação no sistema de saúde. ${ }^{(35)}$

Semelhante ao HLQ, a ferramenta HeLMS também é um questionário de autorrelato, dividido em oito domínios - totalizam 29 perguntas e foi validado em pessoas portadoras de doenças crônicas. ${ }^{(36)}$ Cinco dos domínios do HeLMS se concentram nas habilidades do indivíduo (compreensão das informações de saúde, acesso aos serviços de saúde, comunicação com profissionais de saúde, ser proativo e uso das informaçôes de saúde) e, três dos domínios, em fatores mais amplos, como atitudes, apoio social e fatores socioeconômicos. ${ }^{(19)}$

Ressalta-se que o único instrumento devidamente traduzido para o português do Brasil, adaptado transculturalmente e validado é uma adaptação do REALM, cuja denominação foi modificada para Short Assessment of Health Literacy for Portuguesespeaking Adults (SAHLPA). ${ }^{(34)}$ Embora a ferramenta S-TOFHLA tenha sido traduzida e adaptada, ainda não foi validada, o que é requerido para o seu uso. ${ }^{(37)}$

Considerando os achados na literatura, torna-se importante ressaltar que outras características foram avaliadas concomitantemente ao LFS, como a adesão medicamentosa em pacientes transplantados renais, ${ }^{(17)}$ a qualidade de vida dos que são submetidos a HD, ${ }^{(18)}$ a avaliação dos aspectos cognitivos através do Miniexame do Estado Mental ${ }^{(21,28)}$ e parâmetros laboratoriais, como o fósforo e albumina sérica, buscando verificar a associação da capacidade de compreensão com o seguimento da dieta adequada. ${ }^{(18,28)}$

Os instrumentos encontrados apresentam boas propriedades psicométricas, apesar de a maioria dos pesquisadores ter avaliado a confiabilidade interna pelo alfa de Cronbach - que pode ser influenciado pelo número de itens da ferramenta. ${ }^{(38)}$ Entretanto, é necessário transcender a avaliação numérica e refletir sobre o significado complexo do LFS e qual seria o instrumento capaz de mensurá-lo.
Destaca-se a originalidade deste estudo, visto que é a primeira revisão de literatura sobre este tema. Embora a pesquisa dos artigos científicos tenha sido realizada também na língua inglesa, considerada a mais prevalente na literatura científica, considera-se como uma limitação o fato da utilização de apenas três idiomas para a inclusão de artigos, podendo não ter sido incluídos estudos realizados em outras línguas.

\section{Conclusão}

Há diferentes instrumentos que avaliam o grau de Letramento Funcional em Saúde, entretanto não há nenhum que esteja validado no Brasil, para a população em terapia renal substitutiva com exceção dos transplantados renais. Apesar disto, os resultados da aplicação de algumas dessas ferramentas podem ser comparados nessa população, porque, em geral, avaliam aspectos semelhantes, como reconhecimento, leitura e pronúncia de palavras utilizadas nos serviços de saúde. Embora as propriedades psicométricas de todas as ferramentas identificadas nessa revisão sejam aceitáveis, indicando sua confiabilidade e validade, recomendase a tradução, adaptação transcultural e validação para o português do Brasil, daqueles instrumentos que avaliem aspectos abrangentes dos pacientes especialmente em terapia renal substitutiva, como seu autogerenciamento e capacidade de compreensão das informaçôes, objetivando a otimização de seu tratamento.

\section{Agradecimentos}

À Coordenação de Aperfeiçoamento de Pessoal de Nível Superior (CAPES; bolsa de mestrado), para a autora Késia Tomasi da Rocha.

\section{Referências}

1. Evans PD, Taal MW. Epidemiology and causes of chronic kidney disease. Medicine (Baltimore). 2015;43(8):450-3.

2. Webster AC, Nagler EV, Morton RL, Masson P. Chronic kidney disease. Lancet. 2017;389(10075):1238-52. 
3. Couser WG, Remuzzi G, Mendis S, Tonelli M. The contribution of chronic kidney disease to the global burden of major noncommunicable diseases. Kidney Int. $2011 ; 80(12): 1258-70$.

4. Sesso RC, Lopes AA, Thomé FS, Lugon JR, Martins CT. Brazilian chronic dialysis survey 2016. J Bras Nefrol. 2017;39(3):261-6.

5. Fraser SD, Roderick PJ, Casey M, Taal MW, Yuen HM, Nutbeam D. Prevalence and associations of limited health literacy in chronic kidney disease: a systematic review. Nephrol Dial Transplant. 2013;28(1):129-37.

6. Santos LT, Mansur HN, Paiva TF, Colugnati FA, Bastos MG. Letramento em saúde: importância da avaliação em nefrologia. J Bras Nefrol. 2012;34(3):293-302.

7. Weiss BD, Mays MZ, Martz W, Castro KM, DeWalt DA, Pignone MP, et al. Quick assessment of literacy in primary care: the newest vital sign. Ann Fam Med. 2005;3(6):514-22.

8. Soares M. Letramento e alfabetização: as muitas facetas. Rev Bras Educ. 2004;25(25):5-17.

9. UNESCO Expert Meeting on Aspects of Literacy Assessment Aspects of literacy assessment: topics and issues from the UNESCO expert Meeting [Internet]. Paris: UNESCO; 2005. [cited 2019 Nov 3]. Available from: http://unesdoc.unesco.org/images/0014/001401/140125eo.pdf

10. Pashaki MS, Eghbali T, Niksima SH, Albatineh AN, Gheshlagh RG. Health literacy among Iranian patients with type 2 diabetes: A systematic review and meta-analysis. Diabetes Metab Syndr. 2019;13(2):1341-5.

11. Wong KK, Velasquez A, Powe NR, Tuot DS. Association between health literacy and self-care behaviors among patients with chronic kidney disease. BMC Nephrol. 2018;19(1):196.

12. Tokuda Y, Doba N, Butler JP, Paasche-Orlow MK. Health literacy and physical and psychological wellbeing in Japanese adults. Patient Educ Couns. 2009;75(3):411-7.

13. Kalichman SC, Ramachandran B, Catz S. Adherence to combination antiretroviral therapies in HIV patients of low health literacy. J Gen Intern Med. 1999;14(5):267-73.

14. Baker DW, Wolf MS, Feinglass J, Thompson JA, Gazmararian JA, Huang J. Health literacy and mortality among elderly persons. Arch Intern Med. 2007;167(14):1503-9.

15. Whittemore $R$, Knafl $K$. The integrative review: updated methodology. J Adv Nurs. 2005;52(5):546-53.

16. Dal Sasso MK, Campos PS, Galvão CM. Revisão integrativa: método de pesquisa para a incorporação de evidências na saúde e na enfermagem. Texto Contexto Enferm. 2008;(17):758-64

17. Demian MN, Shapiro RJ, Thornton WL. An observational study of health literacy and medication adherence in adult kidney transplant recipients. Clin Kidney J. 2016;9(6):858-65.

18. Dodson S, Osicka T, Huang L, McMahon LP, Roberts MA. Multifaceted assessment of health literacy in people receiving dialysis: associations with psychological stress and quality of life. J Health Commun. 2016;21(Suppl 2):91-8.

19. Lambert K, Mullan J, Mansfield K, Lonergan M. A cross-sectional comparison of health literacy deficits among patients with chronic kidney disease. J Health Commun. 2015;20(2 Suppl 2):16-23.

20. Shih $\mathrm{CL}$, Chang TH, Jensen DA, Chiu CH. Development of a health literacy questionnaire for Taiwanese hemodialysis patients. BMC Nephrol. 2016;17(1):54.

21. Cavanaugh $\mathrm{KL}$, Osborn $\mathrm{CY}$, Tentori $\mathrm{F}$, Rothman RL, Ikizler TA, Wallston KA. Performance of a brief survey to assess health literacy in patients receiving hemodialysis. Clin Kidney J. 2015;8(4):462-8.
22. Kazley AS, Hund JJ, Simpson KN, Chavin K, Baliga P. Health literacy and kidney transplant outcomes. Prog Transplant. 2015;25(1):85-90.

23. Kazley AS, Jordan J, Simpson KN, Chavin K, Rodrigue J, Baliga P. Development and testing of a disease-specific health literacy measure in kidney transplant patients. Prog Transplant. 2014;24(3):263-70.

24. Escobedo W, Weismuller P. Assessing health literacy in renal failure and kidney transplant patients. Prog Transplant. 2013;23(1):47-54.

25. Jain D, Sheth H, Green JA, Bender FH, Weisbord SD. Health literacy in patients on maintenance peritoneal dialysis: prevalence and outcomes. Perit Dial Int. 2015;35(1):96-8.

26. Green JA, Mor MK, Shields AM, Sevick MA, Arnold RM, Palevsky PM, et al. Associations of health literacy with dialysis adherence and health resource utilization in patients receiving maintenance hemodialysis. Am J Kidney Dis. 2013;62(1):73-80.

27. Green JA, Mor MK, Shields AM, Sevick MA, Palevsky PM, Fine MJ, et al. Prevalence and demographic and clinical associations of health literacy in patients on maintenance hemodialysis. Clin J Am Soc Nephrol. 2011;6(6):1354-60.

28. Cavanaugh KL, Wingard RL, Hakim RM, Eden S, Shintani A, Wallston $\mathrm{KA}$, et al. Low health literacy associates with increased mortality in ESRD. J Am Soc Nephrol. 2010;21(11):1979-85.

29. Lai AY, Ishikawa H, Kiuchi T, Mooppil N, Griva K. Communicative and critical health literacy, and self-management behaviors in end-stage renal disease patients with diabetes on hemodialysis. Patient Educ Couns. 2013:91(2):221-7.

30. Brice JH, Foster MB, Principe S, Moss C, Shofer FS, Falk RJ, et al. Single-item or two-item literacy screener to predict the S-TOFHLA among adult hemodialysis patients. Patient Educ Couns. 2014;94(1):71-5.

31. Adeseun GA, Bonney CC, Rosas SE. Health literacy associated with blood pressure but not other cardiovascular disease risk factors among dialysis patients. Am J Hypertens. 2012;25(3):348-53.

32. Galvão CM. Níveis de evidência [editorial]. Acta Paul Enferm. 2006;19(2):vi.

33. Dageforde LA, Petersen AW, Feurer ID, Cavanaugh KL, Harms KA, Ehrenfeld JM, et al. Health literacy of living kidney donors and kidney transplant recipients. Transplantation. 2014;98(1):88-93.

34. Apolinario D, Braga RC, Magaldi RM, Busse AL, Campora F, Brucki S, et al. Short assessment of health literacy for Portuguese-speaking adults. Rev Saude Publica. 2012;46(4):702-11.

35. Osborne RH, Batterham RW, Elsworth GR, Hawkins M, Buchbinder $\mathrm{R}$. The grounded psychometric development and initial validation of the Health Literacy Questionnaire (HLQ). BMC Public Health. 2013;13(1):658.

36. Jordan JE, Buchbinder R, Briggs AM, Elsworth GR, Busija L, Batterham $\mathrm{R}$, et al. The health literacy management scale (HeLMS): a measure of an individual's capacity to seek, understand and use health information within the healthcare setting. Patient Educ Couns. 2013;91(2):22835

37. Carthery-Goulart MT, Anghinah R, Areza-Fegyveres R, Bahia VS, Brucki SM, Damin A, et al. Performance of a Brazilian population on the test of functional health literacy in adults. Rev Saude Publica. 2009;43(4):631-8.

38. Souza AC, Alexandre NM, Guirardello EB, Souza AC, Alexandre NM, Guirardello EB. Propriedades psicométricas na avaliação de instrumentos: avaliação da confiabilidade e da validade. Epidemiol Serv Saude. 2017;26(3):649-59. 